\title{
Agile Strategic Information Systems based on Axiomatic Agent Architecture
}

\author{
Babak Akhgar \\ C3RI, Sheffield Hallam \\ University,UK \\ Sheffield S1 1WB, Sheffield Hallam \\ University, UK \\ Tel/Fax: +44114225 5555 \\ B.Akhgar@shu.ac.uk
}

\author{
Esmaeil Salahi Parvin \\ Management School, University of \\ Tehran, Iran \\ Jalal-Al-Ahmad Highway, Pol-e-Nasr, \\ Management School, University of \\ Tehran, Tehran, Iran \\ Tel/Fax: +982161117732 \\ e.salahiparvin@ut.ac.ir
}

\author{
Mohammad Hussein Sherkat \\ Management School, University of \\ Tehran, Iran \\ Jalal-Al-Ahmad Highway, Pol-e-Nasr, \\ Management School, University of \\ Tehran, Tehran, Iran \\ Tel/Fax: +982161117732 \\ sherkat@ut.ac.ir
}

\begin{abstract}
Strategic decisions to be made by Strategic Information systems in uncertain environments are considered as the main concern of organizations to achieve differentiating advantages. Architecting such a Strategic Information System (SIS) in which strategic decisions are made continuously can be well performed by employing an axiomatic design approach. The main advantage of axiomatic design approach relies in its potential to determine basic constituents of an agent based SIS. People may decide differently in the same situation not because they are logical but because they sometimes decide emotionally. Regarding emotional characteristics of human being, architecting an SIS based on emotional agents has been proposed in a model. The model contributes in strategic decision making using axiomatic design theory. The main advantage of employing axiomatic design theory is to consider critical points such as emotional decision making and flexibility which results in agile SIS.
\end{abstract}

Architectural design, design theory, system(s) design, Multi Agent Systems, decision making

\section{INTRODUCTION}

The role of information systems (ISs) has evolved during years. The original conception appears in automation of existing manual and pre-computer mechanical processes. This was quickly followed by the rationalization and integration of systems. In both of these forms, IS was regarded primarily as an operational support tool, and less important as a service to management. During the 1980 s, an additional potential was discovered. It was found that, in some cases, information technology (IT) had been critical to the implementation of an organization's strategy. The dominant sense in which the term is used is that a strategic information system (SIS) is an information system which supports an organization in fulfilling its business goals (Shirazi and Soroor, 2007).

Strategic information systems focus on the use of information systems and information technology in the strategic management process in businesses. The emphasis is on the strategic view of IS and IT and their impact on organizational strategy. A Strategic information system concerns itself with systems whose importance to the organization extends beyond merely assisting it to perform its existing functions efficiently, or even just efficiently (Shirazi and Soroor, 2007). The topic of 'strategic information systems' is concerned with systems which contribute significantly to decision making in the organization.

One of the important aspects of the use of strategic information systems in organizations is their impact on increasing the level of agility in decision-making process.

By comparing the level of agility in human and system decision making processes, it can be considered that emotion is the main factor in increasing the agility of mentioned process. Perhaps this factor can be considered as the most 
important factor in creating effective human decision-making process.

An Agile decision-making process is characterized by its ability to change significantly the manner in which the business is carried out via changes to goals, processes, products or environmental relationships due to environmental changes (Turban et al., 2001).

Architecting an agent based strategic information system in which strategic decisions are made agilely is the authors' main concern. Axiomatic design theory enjoys major characteristics to lead the design process in which basic constituents can be determined to help the firm survive in an uncertain and competitive environment and to acquire competitive advantages against business rivals. These constituents are critical to make strategic decisions. Axiomatic design has been used here to establish an SIS agent-based model based on its characteristics. Axiomatic design employing a zigzagging process starts to map the functional requirements in the functional domain to the design parameters constituent in the final architecture of an agent based SIS. It should be noted that identifying major agents which figure the strategic decision making process using an axiomatic design theory is performed regarding their main interaction principles including communication, negotiation and coordination. The way these agents are interacting regarding emotional factors is considered regarding interaction mechanisms and functions such as policy and learning.

Emotional agents are new ideas in multi-agent systems that are used to increase agility in decision-making process in strategic information systems. The proposed model has regarded the emotionality as a new communication factor among the experiences.

This article is arranged in five sections starting with the introduction and following by second section that introduces axiomatic design theory. Emotional agents and their role in SIS are introduced in the third part. The article goes further in Part four illustrating a model for SIS based on Axiomatic Architecture. Last but not the least the final part deals with the results and conclusions.

\section{AXIOMATIC DESIGN THEORY}

The ultimate goal of Axiomatic Design (AD) is to establish a scientific basis for design and improve design activities by providing the designer with a theoretical foundation based on logical and rational thought processes and tools (Suh, 2001). In accomplishing this goal, the $A D$ provides a systematic search process through the design space to minimize the random search process and determine the best design solution among many alternatives. $A D$ theory and principles are introduced by Suh (Suh, 1997) and its application in designing products, systems, organizations and softwares appeared in the literature. AD principles are also used in the design of quality systems (Suh, 1995) and general system design (Suh, 1997) providing a manufacturing system design.

\subsection{Axiomatic Design principles}

The most important concept in AD is the existence of the design axioms. The first design axiom is known as the Independence Axiom and the second axiom is known as the Information axiom. They are stated as following (Kulak et al., 2005a):

- Axiom1.The Independence Axiom: Maintain the independence of functional requirements.

- Axiom2.The Information Axiom: Minimize the information content.

The Independence Axiom states that the independence of functional requirements (FRs) must always be maintained, where FRs are defined as the minimum set of independent requirements that characterize the design goals (Suh, 1990). In the real world, engineers tend to tackle a complex problem by decomposing it into sub-problems and attempting to maintain independent solutions for these smaller problems. This calls for an effective method that provides guidelines for the decomposition of complex problems and independent mappings between problems and solutions. The Information Axiom states that among those designs that satisfy the Independence Axiom, the design that has the smallest information content is the best one (Suh, 2001).

Axiomatic design theory provides a systematic methodology and a hierarchical approach for the engineering design process. Axiomatic Design process is implemented based on three major concepts: domains, hierarchies and zigzagging. Suh (Suh, 1998) identified four domains including (1) customer domain which provides Customer Needs (CN) or customer attributes (CA) (2) functional domain providing Functional Requirements (FR) (3) physical domain providing Design Parameters (DP), and the (4) process domain, providing Process Variables (PV). The Axiomatic design process is centred around the satisfaction of functional requirements which are defined as the minimum set of independent requirements characterizing the component design goals (Kulak et al., 2005b).

The needs that the customer is looking for characterize the customer domain in a product, 
process or system (CA). The customer needs can be specified in the functional domain employing terms of functional requirements. Functional Requirements can be specified using minimum collection of Requirements which are independent and characterize objectives of the design process for a specific need completely (Suh, 1990).

Functional Requirements (FRs) are to be independent of each other on any level in the functional hierarchy. Design parameters (DPs) indicate the physical attributes or objects that will be produced by the design process to meet the required FRs in the functional domain. The actions which are required to satisfy customer needs are described by Functional Requirements and physical entities that have to be made to meet their Functional Requirements are determined by the design parameters (DPs). To produce the product that is specified in terms of DPs, a process is evolved which is specified by process variables (PVs). These process variables are determined in the process domain. The design procedure is taken by a repetitive process between what the designer wants to achieve and the way he achieves it (Suh, 1990). Figure 1 illustrates the four mentioned domains in the axiomatic design process. The second concept of axiomatic design, also consistent with Herbert Simon's, is hierarchical decomposition process for requirements analysis. According to Simon, an important approach to solving complex problems is to divide the problems into simpler parts, solve them, and integrate into the solution (Nucci Franco and Batocchio, 2001). Axiomatic Design decomposes the problems into simpler parts since it has a top-down approach. The third concept of Axiomatic Design is zigzagging. Instead of decomposing only the FR domain thoroughly, independent of any other domain, zigzagging allows a parallel decomposition of all four domains (Togay et al., 2006).

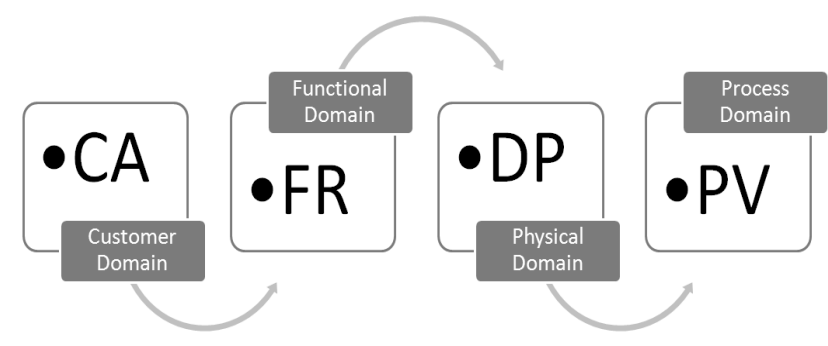

Figure 1: The four domains in the axiomatic design process

The way axiomatic design maps between functional domain and physical domain help the designer to start from a point in the domain of functional requirements and zigzag to the domain of physical parameters to meet the functional requirements is determined by customer attributes. Then by continuing the zigzagging process from physical domain to functional domain, the functionalities required by those physical parameters are identified. This mapping process particularly helps the system designer to identify all functional requirements and physical parameters of an agent based SIS in which strategic decision making is considered as a major process to help the system acquire strategic competitiveness over the rivals. All of the functionalities included in an agent based SIS are determined using this hierarchical zigzagging process between physical and functional domains.

\section{EMOTIONAL AGENTS IN SIS}

Decision-making processes are usually categorized into three levels: strategic decisions, tactical decisions and operational decisions. Strategic decision making processes are considered as one of the core processes of Strategic information systems which specify the objectives and resources needed to realize them in companies. Reviewing early work in the field of Strategic decision making, four major characteristics are attributed to it: Irregularity, Complexity, IIIstructured problems and Uncertain and imprecise knowledge (Pinson and Moraitis, 1997). The problems which are indicating mentioned attributes have employed solutions based on cooperation, coordination and negotiation between several agents. In Strategic information systems; it is common to assume that decisions are made based on clear rules and guidance, a table or graph. This approach hardly has the ability to model circumstances in which decisions are taken by humans. People may decide differently in the same situation not because they are logical but because they sometimes decide emotionally. Rationality is independent of the people, so it is comprehensible for all, that is, the decision maker is always trying to maximize its direct benefit by making decisions. In contrast, emotionality is very personal and often leads to inexcusable decisions.

Sloman and Croucher (Sloman and Croucher, 1981) detailed naive Psychology achievements to conclude that "because of the need to cope with the changing and somewhat unpredictable world, it is likely that any intelligent system with different motivations and limited power will be emotional. Therefore, the belief that emotions and reason are of distinct species is mistaken. They have identified some limitations on intelligent systems as "recognition often requires the use of description," "Motivation is not static", "environment is not static", "the complexity of the environment often leads to false beliefs, plans, and actions", and "different motivations in the same individual may be inconsistent". 
Sloman (2004) suggests that "what are usually called emotions are to somehow fuzzy subsets of a larger class of states and processes that can be raised as interactions between the different mechanisms in the architecture (Sloman, 2004). Decision-makers change their behaviours regarding information they receive. These objects act in different informational and behavioural situations as executives of the result of processing information and so are called agents whose Motivating factors are independent. Each agent type has a set of related collection of rule-sets. Each rule-set is a collection of condition-action rules that act through interaction 'databases' or operating with internal memories. So, a rule set may be concerned with the interpretation of lowlevel sensory information, other productive motivators in response to the formation of new beliefs, the importance of assessment in relation to other motivators, another plan, and so on. Within an agent, learning or development processes, may change individual rules or introduce new rule sets or interaction between the rule sets, for example modified by adding new communication channels (Davis et al., 1995). Macas et al., argue that effective decision-making depends on the intensity of the emotions and fundamental mechanisms and that alternative courses of action in a decisionmaking problem are emotionally (somatic) marked as good or bad (Macas et al., 2001).

Most independent agents are located in social areas and need to interact with other agents (both human and artificial) to solve their problems. This interaction purposes many potential decision making functions that can be selected to make the choice. Such a function of an individual agent in the success will be different of the overall system in which it is located. Therefore Kalenka and Jennings suggested agents' decision making functions to know the properties and characteristics (Kalenka and Jennings, 1997).

Reviewing the previous work, there are multiple approaches for defining Policy Function of the agents. Based on the definition of Wooldridge and Jennings (1995) policy function is a function that maps the pattern profile of each entity to the quantitative amount (Wooldridge and Jennings, 1995). Sutton and Barto (1998) believe that the policy function is any function that enables the agents to map the environment to a point in decision space (Sutton and Barto, 1998). Peshkin (2003) discussed about the optimal policy function to search in a society of autonomous agents based on game theory, although his main focus was on the gradient method (Peshkin, 2003).

One of the methods of interaction establishment between agents in Multi Agent Systems (MASs) is market mechanism (Wurman, 1999, Wurman et al.,
2001). Such a method devised on the basis of Adam Smith's theory on free market movement to a suitable balance point. According to market mechanism principles, each agent with regard to its capabilities, wealth and utility function for goods that are offered in the market proposes a price and of course it will be the winner provided that it purchases the goods (services) in a situation that there is no higher offer. The fact that the seller wants to sell his goods for the maximum possible prices is absolutely logical; of course, it is expected that rich agents' tendency toward purchasing low quality products should be less than poor agents that are aware of their chance of gaining quality products. Along with the mechanism described above, negotiation and bargaining mechanism between agents can also be used as a complement mechanism which of course requires defining special communicative protocols between agents respective to chain agents.

Interaction mechanism between agents can also be studied as a game theory perspective (Cicirello, 2001). (MASs) are closely associated with game theory. Each agent plays as an actor that decides to maximize its goals for the long term.

According to John Nash studies in the games theory, it can be shown that this game certainly has a point of Nash's equilibrium point where one of the players does not want to be out of the balance. Nash's Equilibrium points are certainly optimal Pareto's points in the system, but there is no guarantee that this point is based on the system's optimum point.

Alternative courses of action in decision-making problems are emotionally (somatic) specified as good or bad. The emotional symptoms not only guide the decision-making process, but also leave positive options to be considered for further review. Considering the role of Strategic decision making processes in evolving the objectives of the processes of Strategic information systems in acquiring competitive advantage, it should be noted that the field of artificial intelligence has largely ignored the use of emotions and intuition to guide reasoning and strategic decision making. Velasquez 's (1998) contribution shows "how the drives, emotions and behaviours can be integrated into strong agent architecture, using some of the mechanisms of emotion to get the memories of emotional experiences that serve as biasing mechanisms, while making decisions during the operation-selection process"(Velasquez, 1998).

Santos et al. proposed architecture for a ubiquitous group decision support system able to support persons in group decision processes. The system considers the emotional factors of the intervening 
participants, as well as the argumentation between them.

The flexible agent integration architecture is originated from emotions, behaviours and emotions. Emotions as the main motivation system influence the way behaviours are selected and controlled. Here it is tried to show the primary mechanism of how feelings can serve such building blocks to achieve emotional memories such as a biasing mechanism in the process of decision making and action selection.

(El-Nasr et al., 1998) rely on Demasio's proposed model that emotions lead an active role in conducting the decision-making process by providing a selection mechanism for removing bad alternative options.

Later, (El-Nasr et al., 2000) developed a model using fuzzy logic representation to map events and observations to emotional states. Their proposed Model also includes several inductive learning algorithms for learning patterns of events, associations among objects, and their expectations (Gmytrasiewicz and Lisetti, 2002).

Emotional agents interact within the structure of an agent based SIS in which strategic decision making is performed based on interaction mechanisms such as negotiation, communication and coordination. These mechanisms are mainly influenced by emotional status of each agent during data gathering, decision making and decision implementation activities followed by the agents. So to identify the structure of an agent based architecture regarding axiomatic design theory, it is the role of emotionality and interaction parameters which is to be followed particularly by the designer.

\section{SIS MODELLING BASED ON AXIOMATIC AGENT BASED ARCHITECTURE}

The organization of an SIS can be established regarding the holonic paradigm which reminds that any decisive structure with a minimum limit of stability and cohesion can be arranged hierarchically. Employing this concept of holonic systems, it is possible to design the SIS based on agents using three major categories of them: awareness, decision making and implementing agents. The total synergy of these agents is improved based on their ongoing interaction and the way they are organized. Organizing the total structure of the SIS can be routed through determining the functional requirements (FRs) of the constituents. Mechanised actions and changing strategies are shaped in agent based system structures. Top level agents have the most capacity to change their FRs regarding the changing environment while bottom-level agents have less capacity and are stiffer. This hierarchical attribute is compatible with a typical organizational structure in which the strategic level of the system deals with high level uncertainty and operational level of the system deals with low level uncertainty. The communication parameters between agents are one of the important attributes which include protocols, languages and levels of interaction. An overall agent must be able to decide what to do in every situation. So it has to recognize the operational options and evaluate them based on attributes recognised during the decision process.

After determining functional requirements related to different agents, design parameters (DPs) associated with each FR will be identified using a dynamic mapping function. This function is determined depending on the conditions and limitations of the decision making situation. Figure 2 shows the hierarchy of the FRs established in a changing environment and Figure 3 shows the hierarchy of the DPs identified using a mapping function. Accepting the idea of finding a dynamic mapping function to identify DPs, it could resulted in conditions in which two structurally similar agents (in any level) will never have similar decision making styles. It is because of the changing environment and emotionally changing agents which will change their DPs based on the required FRs.

Finding a function to map from a functional domain to a physical domain is possible based on an indirect probe in decision space. Using the agent concept in this model facilitates the decomposition of problem specifications in a predefined framework and also defining the mapping function of functional domain to physical domain based on policy function of the agents.

The agility of the designed SIS directs the designer to constitute the architecture agents enjoying awareness, decision making and decision implementation functionalities in the physical domain (Figure 3).

Awareness, emotional decision making and implementation functionalities are obtained by including abilities in the agent's design parameters in the physical domain. The process of axiomatic design zigzagging continues to determine all required functionalities in the functional domain and all design parameters in the physical domain.

\subsection{AWARENESS}

Constituting an aware agent in physical domain results in mapping onto functionalities such as: Data communication, Data processing and analytic 
report generation. These functionalities (Figure 2) help the designer to include the aware agent in the physical domain. Establishing the above-mentioned functionalities for an aware agent, three design parameters are recognised to be included in the physical domain (Figure 3): data receivers, data processors and report generators.

\subsection{EMOTIONAL DECISION MAKING}

Constituting an emotional decision maker agent in physical domain maps onto six functionalities including: problem generation, CSF recognition, emotional policy function formation, learning, interagent interaction and emotional decision analysis. These functionalities (figure 2) help the designer to include the emotional decision maker in the physical domain. Setting up these functionalities for an emotional decision maker agent, six design parameters will be identified to be included in the physical domain (figure 3): problem generators, CSF identifiers, emotional policy function parameters, learning agent, emotional interaction capacity and emotional thinking mechanisms.

Agents have a tendency to define their policy function to maximize utility which is acquired in transition process from functional domain to physical domain. To frame the above-mentioned process, the agent follows 5 steps:

\subsubsection{Recognising the patterns of the entity in functional level}

The main objective of this step is recognizing the most important attributes of decision making entities which can affect the utility amount from the agent's point of view.

\subsubsection{Manipulation of patterns}

The process of Manipulation of patterns is looking for deletion of dependent parameters of patterns which can influence the decision making process of a system.

\subsubsection{Formalizing a model of agent's policy} function

The agent's policy function could be a variant function, but in order to acquire better results, it can exploit the following attributes:

- The ability to map similar patterns in functional domain to a predetermined level in physical level;

- The ability to exploit pre-existing knowledge in the system. Using the previous results of system decisions and the history of the system's and agent's decisions can facilitate reaching to better decisions;

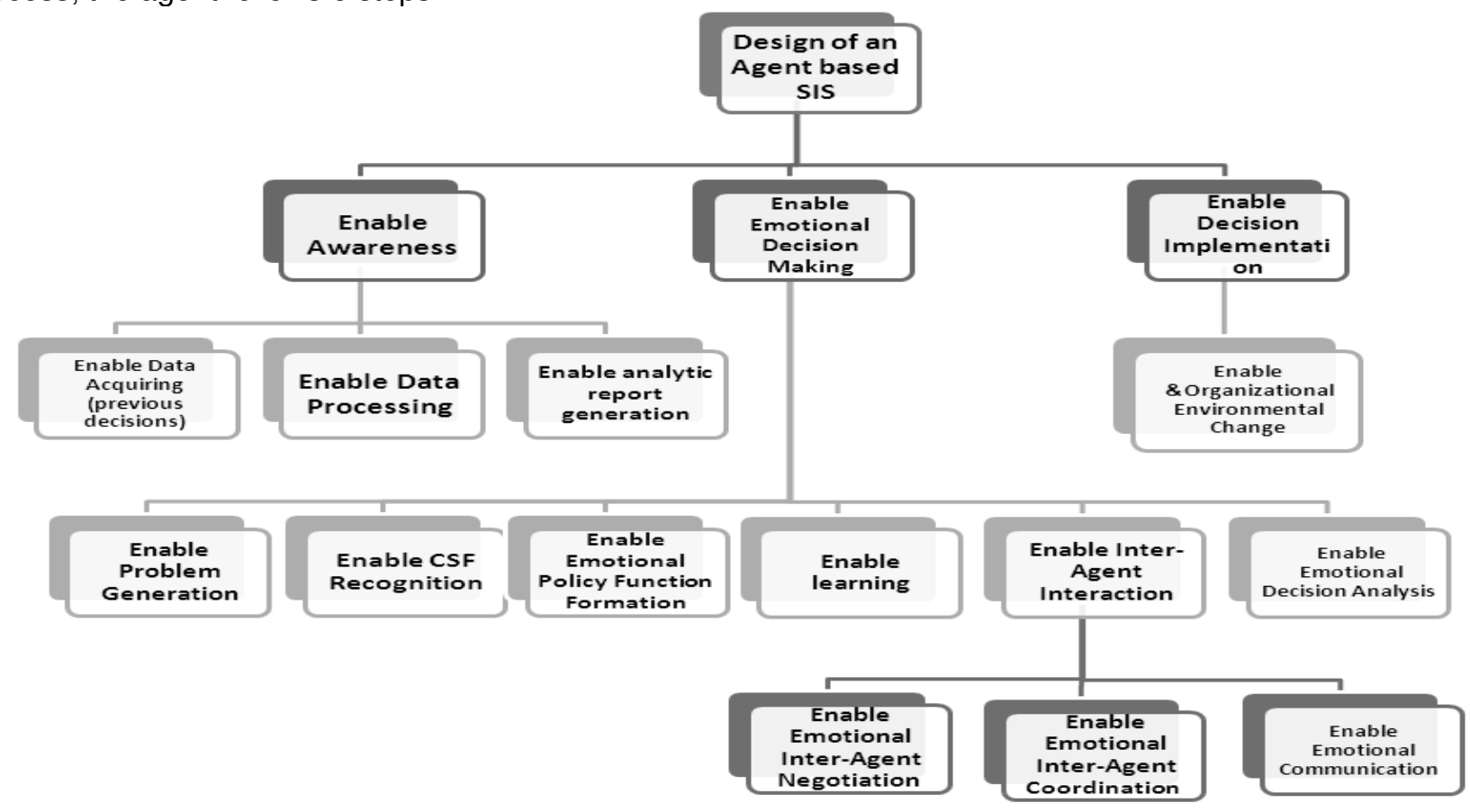

Figure 2: The hierarchy of FRs

- The ability to produce non-linear behaviours and make decisions in the physical domain based on the conditions in functional domain. So the probability of producing directed and linear decisions is increased and the system will not behave according to the contingencies of the environment. 
If the history of the previous decisions is available, fuzzy inference and neural networks will be good choices to estimate the policy function. This selection is because of the abilities of fuzzy inference to use the system knowledge and of neural networks to employ the logs of the system behaviour which can estimate the mapping function. It is noticeable that the policy function is a function of mapping from functional domain to physical domain.

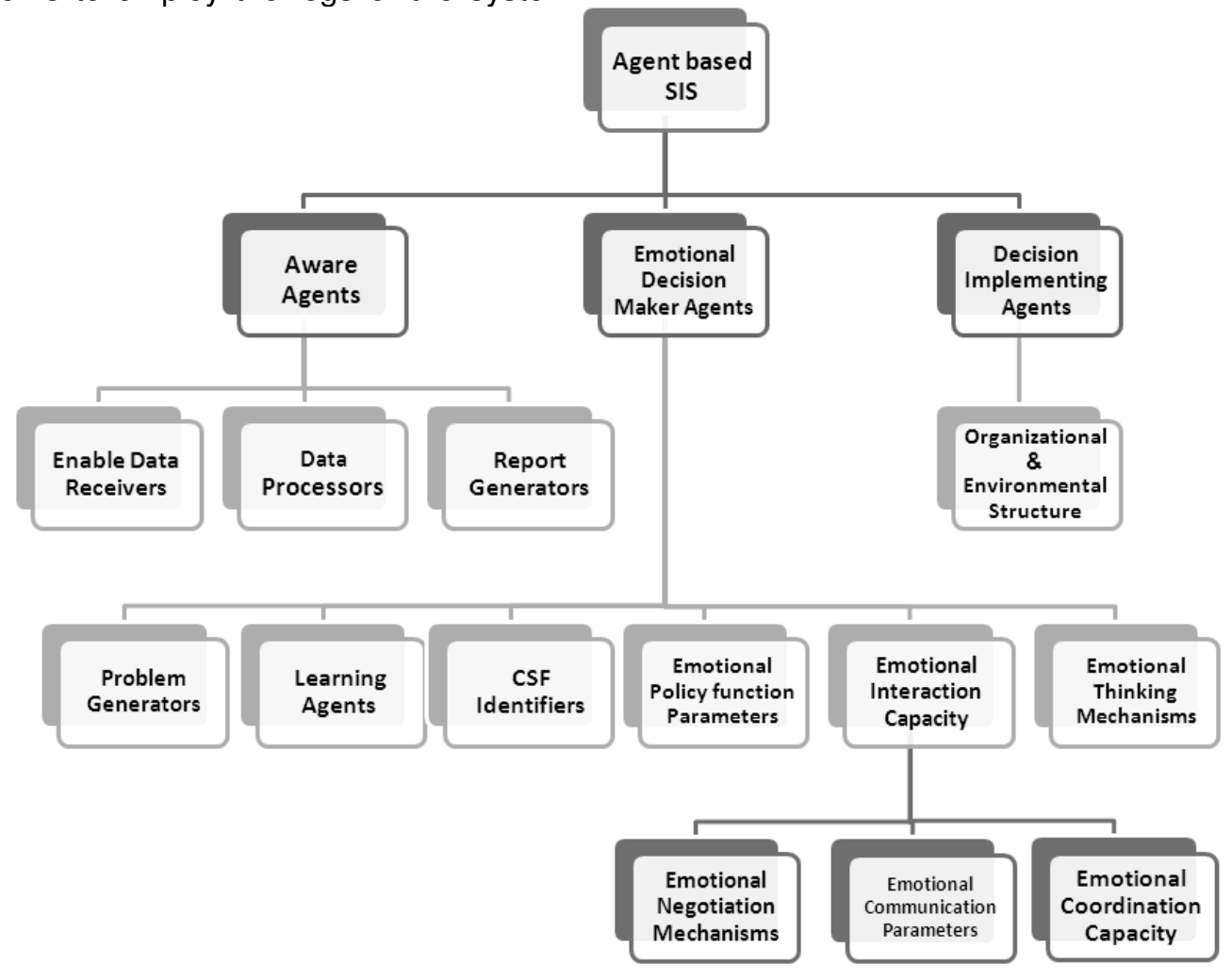

Figure 3: The hierarchy of the DPs

If there is no previous history of the system's decisions, reinforced learning to find the policy function and state-action, matrixes would be useful.

\subsubsection{Design of a mechanism to transform the utility of institutions of functional level to utility of institutions of physical domain}

The utility of institutions in transition from function domain to physical domain is to be protected from influence. If these utilities change during the transition process, the final decision making will be influenced in a wrong way.

\subsubsection{Design of a mechanism to improve the agent's policy function;}

If the policy function enjoys multiple free parameters, it can be configured using a predetermined mechanism to reach better decisions. To search for convenient parameters of the policy function, searching algorithms such as Genetic algorithms and simulated annealing will be useful.

Setting up inter-agent emotional interaction capacity requires identifying multiple functionalities in the functional domain including: emotional coordination, emotional negotiation and emotional communication (Figure 2). So to reach the mentioned functionalities the designer sets to reach several design parameters in the physical domain: Emotional negotiation mechanisms, Emotional communication parameters and Emotional coordination Capacity (Figure 3).

A decision will transfer reward for the decision maker in the long run. Classical economics defines the rules of logic to model the process of society and even the best decisions for the decision maker in every situation. Thus, the results of decisions which are made in the system are dependent on the agent, and the environment. Therefore, it is true to describe the "rational decisions" as "definitive." This approach may fail; however, decisions vary because of different agents and environmental conditions. Building a more general framework for decision making, the personality of the decision maker can be as integrated in to model as the agent's emotional. A rational decision is an optimum solution intended to maximize the reward as a set of constraints. Thus, in most cases, it can be left to a computer to find a solution, if the 
objective function and constraints are defined appropriately using mathematical terms.

\subsection{DECISION IMPLEMENTATION}

Applying the results of the decisions made by the emotional decision maker, there will be some feedback from the environment of the SIS. This environment could both include stakeholders and the rivals of the company. To include the parameter of the decision implementation agent, there will be the functionality of the organization and the environment that can result in the changing of the internal structure of the organization and the transforming of the environment (Figure 2). By Zigzagging this functional parameter regarding the axiomatic design, there will be an organizational and environmental structure in the physical domain (Figure 3).

What an agent gains by a decision which is made may cause changes in the characteristic set of that agent, i.e., its emotions. If the agent is gaining some profit by taking a risk, this gain strengthens the agent's level of accepting risks. This can be interpreted as the reinforcement learning of the agent.

It is important to note that the adjustment time may be different from the personal characteristics of the system change state or environmental conditions. Therefore, the decision maker receives information of the state of the system and a broader environment according to which the decisions are made and the emotional levels are adjusted in different time scale.

Setting up the agents described and regarding the presence of the environmental subjects including stakeholders and rivals, a broad model of the rolling SIS in the company could be achieved. Figure 4 shows a schematic model of strategic information system in which the decision making character is affected by the environment and the rewards. As it can be seen, there are certain kinds of data and information communication between the agents and also between the agents and the environment. Reward is the personal measure of the consequences of decisions made by the agent. Multivariate average function at all levels during each stage determines the reward by the different weights assigned by the agent. Results of the decisions change the state of the system and the environment.

\subsection{The proposed model for the SIS}

The system state may be influenced by other agents. The environment may change unexpectedly. Agents face new circumstances and decide. In fact, various agents in different systems with different rules play a game to maximize their own profits. Their interaction defines the system status at anytime.

Each agent in the proposed model of the rolling SIS enjoys a definite structure which facilitates the process performed by them. These objects in the structure of each agent are acquired regarding the zigzagging process proposed by the axiomatic design theory. Figures 5 and 6 illustrate the internal structure of awareness and decision making agents. Certain tools in decision making agent's structure are modified to include the emotionality factor impressing the decision making process. These structures are established regarding the basic overall structure of the mentioned SIS.

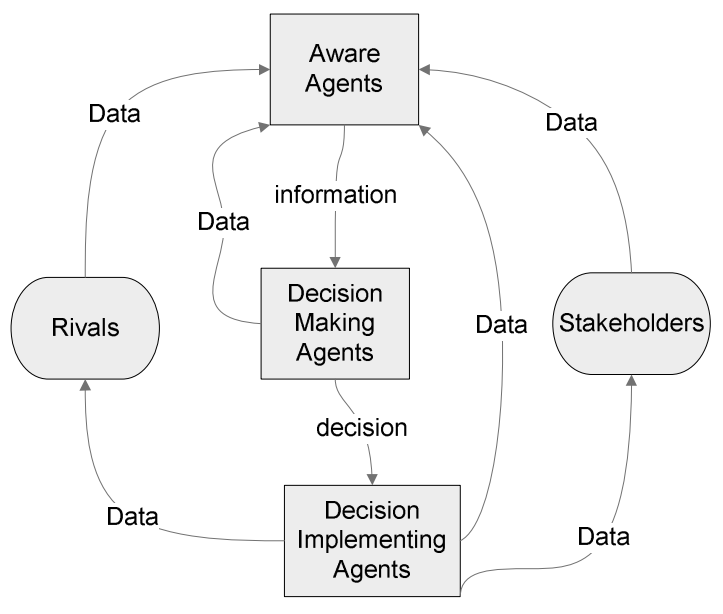

Figure 4: A broad model of the rolling SIS in the company regarding the environment

The process of zigzagging between the functional and physical domains which is proposed by the axiomatic design theory has facilitated the recognition of certain functionalities and design parameters and will help the totality of the system to be survived and evolved in a competitive environment. Using an emotional factor, agents have the tendency to cooperate together and transform the individuality of each agent to the overall object oriented constituents of a bigger system called SIS. There are roles allocated to each agent which illustrate the totality of the functionality of that agent and its respective design parameters. Using this structure, each agent is determined as a centre of fixed and flexible rules and strategies. Their behaviour containing permissions, prohibitions and obligations will be shaped and direct the process of decision making in each moment about the actions. These actions help to acquire long term and mid-term success measures. To reach the flexibility of functionalities for the system, axiomatic design theory makes identifying the required design parameters possible. 


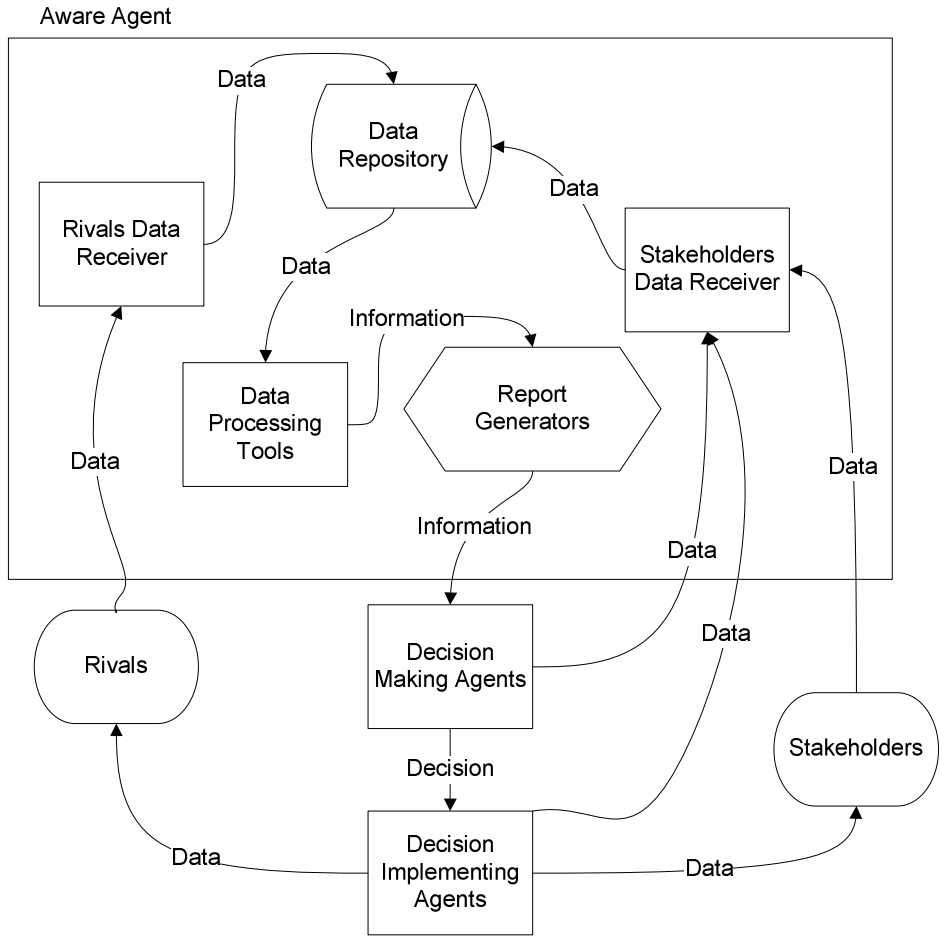

Figure 5: The internal structure of an aware agent acquired by axiomatic design theory

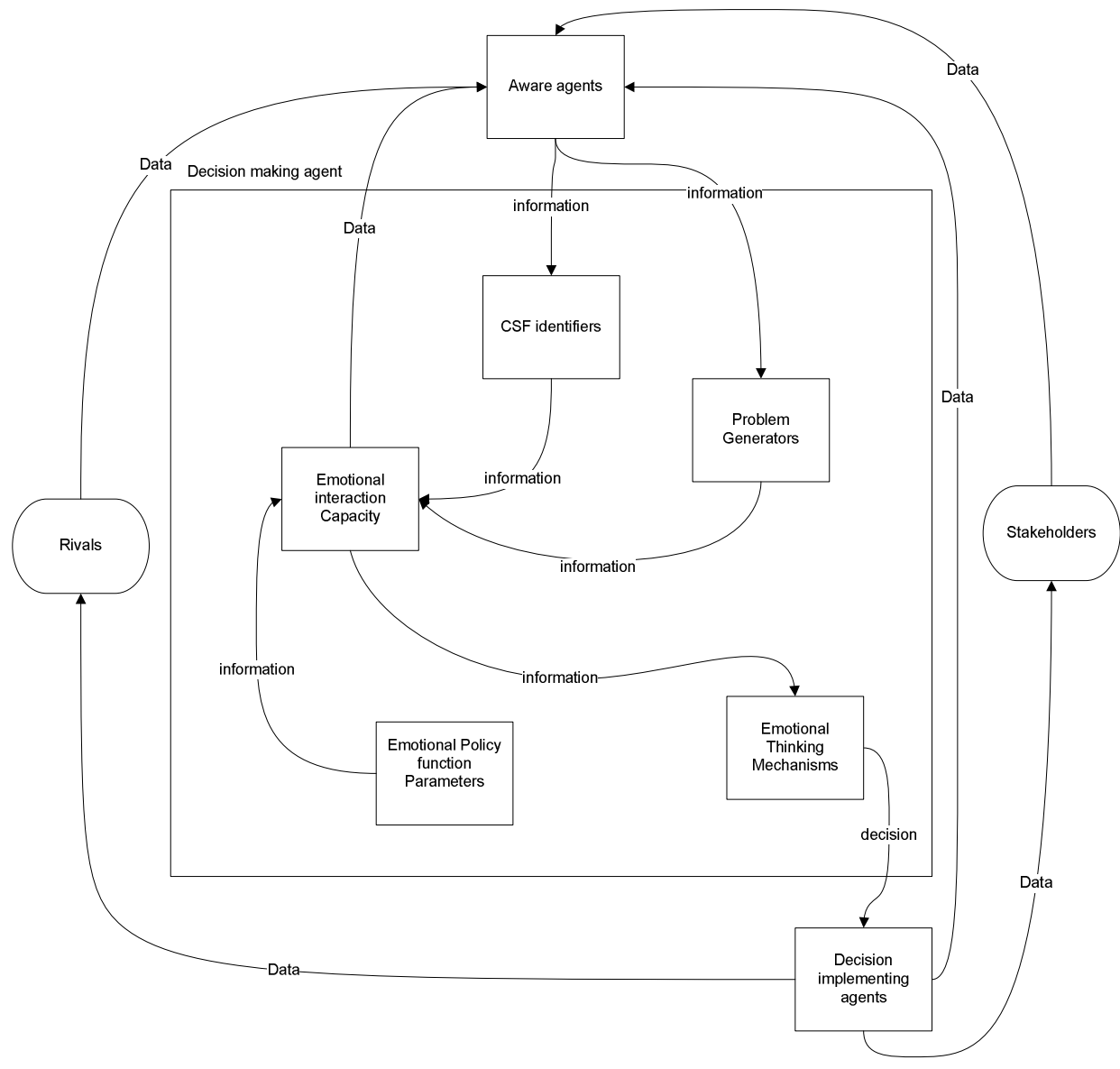

Figure 6: The internal structure of a decision making agent acquired by axiomatic design theory 


\section{CONCLUSION}

Architecting an SIS based on emotional agents who contribute in strategic decision making based on axiomatic design theory has been proposed in the model developed in this article. It should be noted that there are multiple benefits in utilizing these concepts. It makes it possible to enjoy the ability to artifact the SIS architecture and make it support the process of competitive gains against rivals and help the survival of the company.

Considering making strategic decisions in an agent based SIS, we have made use of agent systems which are initiatives of modern artificial intelligent advances and has been developed in order to meet the requirements of the new world while their evolvement is still continuing.

An axiomatic design approach has been employed to support the process of mapping between functional requirements and physical parameters recognized as critical to architect an agent based SIS. This process of zigzagging has supported identifying required functionalities and their respective design parameters which forms the basic architecture of an SIS and supports the process of strategic decision making. Regarding special functionalities and their respective design parameters such as inter-agent interaction mechanisms and emotional policy functions, the proposed architecture enjoys a powerful tool to perform the process of decision making. An Interaction between existing agents has been shaped and similarly, different methods of decisionmaking have been studied and several suggestions have been proposed based on dominant conditions. As it can be seen from previous studies, a limited number of tools are capable of establishing interaction including communication, negotiation and coordination mechanisms between agents and integrating information inside and outside the agents. The proposed model has tried to include three kinds of agents and their respective structures based on axiomatic design theory.

By reviewing the analysis performed in the article, it is obvious that three particular attributes are captured in the proposed model:

(i) Extensive interaction including negotiation, coordination and communication between system agents and with stakeholders and business rivals to increase flexibility;

(ii) The possibility to gather and analyze information and make effective decisions;

(iii) Learning, adaptation and agility;
The proposed model has effectively made it possible to rapidly change the decision-making parameters in an SIS (such as adding or changing decision-making rules) which leads to an increase in agility in the SIS.

\section{REFERENCES}

CICIRELLO, V. A. (2001). A game-theoretic analysis of multi-agent systems for shop floor routing, Pittsburgh, $\mathrm{Pa}$., Carnegie Mellon University, The Robotics Institute.

DAVIS, D., SLOMAN, A. \& POLI, R. (1995). Simulating Agents and Their Environments AISB Quarterly, 34-41.

EL-NASR, M. S., SEIF, M. \& SKUBIC, M. (Year). A Fuzzy Emotional Agent for Decision-Making in a Mobile Robot. In: IEEE International Conference on Fuzzy Systems, 1998.

EL-NASR, M. S., YEN, J. \& IOERGER, T. R. (2000). FLAME - Fuzzy logic adaptive model of emotions. Autonomous Agents and Multi-Agent Systems, 3, 219-257.

GMYTRASIEWICZ, P. J. \& LISETTI, C. L. (2002). Emotions and personality in agent design and modeling. Intelligent Agents Viii: Agent Theories, Architectures, and Languages, 2333, 21-31.

KALENKA, S. \& JENNINGS, N. R. (1997). Socially Responsible Decision Making by Autonomous Agents, Springer Verlag.

KULAK, O., DURMUSOGLU, M. B. \& TUFEKCI, S. (2005a). A complete cellular manufacturing system design methodology based on axiomatic design principles. Computers \& Industrial Engineering, 48, 765-787.

KULAK, O., KAHRAMAN, C., OZTAYSI, B. \& TANYAS, M. (2005b). Multi-attribute information technology project selection using fuzzy axiomatic design. Journal of Enterprise Information Management, 18, 275 - 288.

MACAS, M., VENTURA, R., CUST'ODIO L. \& PINTO-FERREIRA, C. (Year). Experiments with an Emotion-based Agent using the DARE Architecture. In: Symposium on Emotion, Cognition and Affective Computing (AISB'01 Convention), 2001 UK.

NUCCI FRANCO, G. \& BATOCCHIO, A. (Year). Towards an axiomatic framework to support the design of holonic systems. In: Database and Expert Systems Applications, 2001. Proceedings. 
12th International Workshop on, 2001 2001. 654659.

PESHKIN, L. (2003). Reinforcement learning by policy search, [Cambridge, Mass.], Massachusetts Institute of Technology - Artificial Intelligence Laboratory.

PINSON, S. \& MORAITIS, P. (1997). An Intelligent Distributed System for Strategic Decision Making. Group Decision and Negotiation, 6, 77-108.

SHIRAZI, M. A. \& SOROOR, J. (2007). An intelligent agent-based architecture for strategic information system applications. Knowledge-Based Systems, 20, 726-735.

SLOMAN, A. (Year). What are emotion theories about? In, 2004. 128-134.

SLOMAN, A. \& CROUCHER, M. (Year). WHY ROBOTS WILL HAVE EMOTIONS. In, 1981. 197202.

SUH, N. P. (1990). The principles of design, New York, Oxford University Press.

SUH, N. P. (1995). Design and Operation of Large Systems. Journal of Manufacturing Systems, 14, 203-213.

SUH, N. P. (1997). Design of Systems. CIRP Annals - Manufacturing Technology, 46, 75-80.

SUH, N. P. (1998). Axiomatic design theory for systems. Research in Engineering Design-Theory
Applications and Concurrent Engineering, 10, 189209.

SUH, N. P. (2001). Axiomatic design : advances and applications, New York, Oxford University Press.

SUTTON, R. S. \& BARTO, A. G. (1998). Reinforcement Learning I: Introduction, MIT.

TOGAY, C., SUNDAR, G. \& DOGRU, A. H. (Year). Detection of component composition mismatch with axiomatic design. In, 2006. 153-158.

TURBAN, E., MCLEAN, E. R. \& WETHERBE, J. C. (2001). Information technology for management : making connections for strategic advantage, New York, J. Wiley.

VELASQUEZ, J. D. (Year). When robots weep: Emotional memories and decision-making. In, 1998. 70-75.

WOOLDRIDGE, M. \& JENNINGS, N. R. (1995). INTELLIGENT AGENTS - THEORY AND PRACTICE. Knowledge Engineering Review, 10, 115-152.

WURMAN, P. R. (1999). Market structure and multidimensional auction design for computational economies.

WURMAN, P. R., WELLMAN, M. P. \& WALSH, W. E. (2001). A parametrization of the auction design space. Games and Economic Behavior, 35, 304338. 